\title{
STAYING AWARE OF RELEVANT FEEDS IN CONTEXT
}

UBC THOMAS FRITZ

fritz@cs.ubc.ca

\section{MOTIVATION}

Developers face an avalanche of information daily.



Overlooking relevant information results in productivity loss. [1]

\section{APPROACH}

Extending the Information Fragment Model with Dynamic Fragments A feed is a stream of information that changes constantly with new incoming information items. Our approach extends our previous Information Fragment Model [2] with the concept of dynamic information fragments, such as feeds.

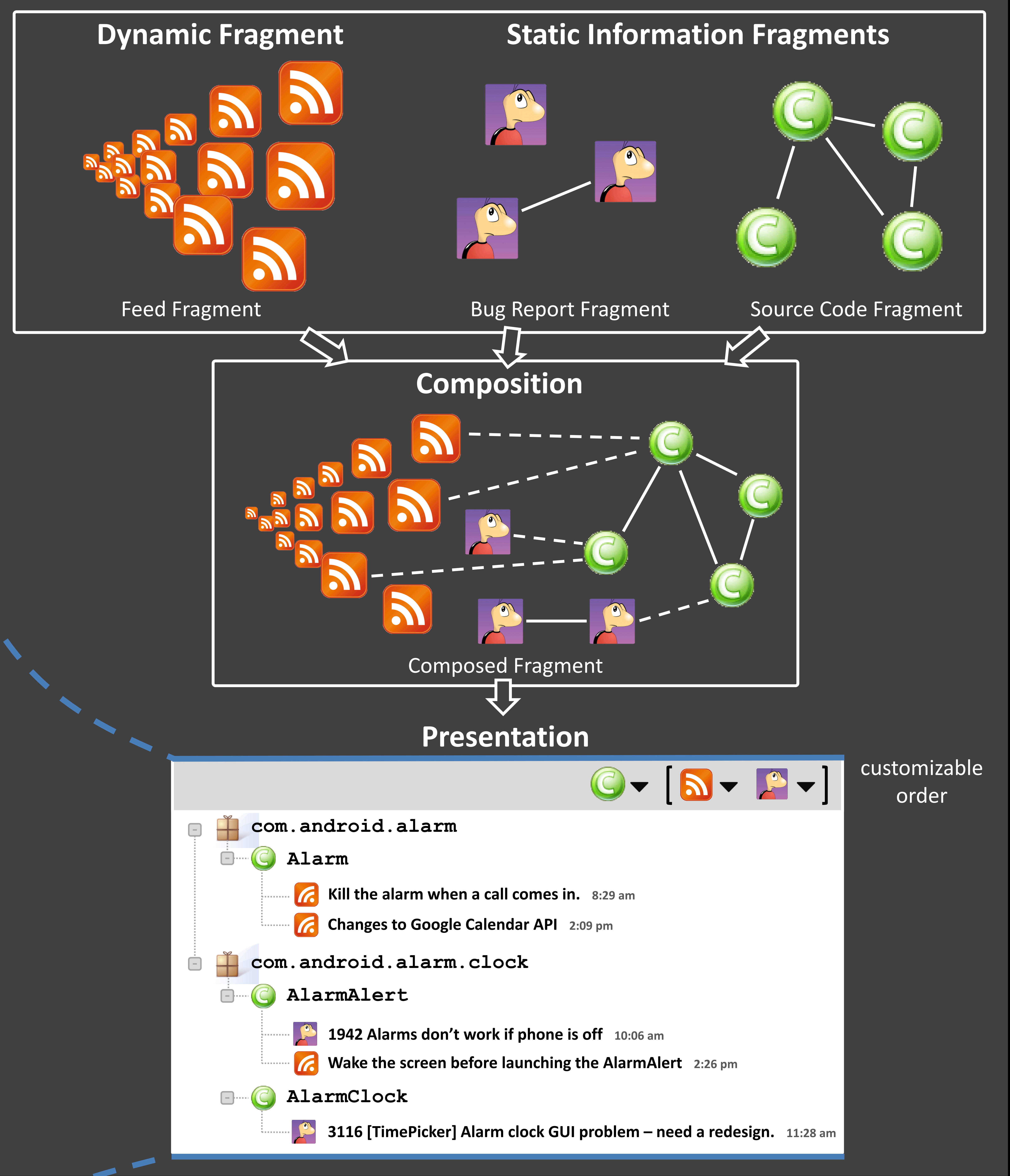

\section{RESULTS \& CONTRIBUTIONS}

A fluid integration of dynamic and static information that provides a context for interpreting feeds and supports various means of ranking and filtering feeds. This allows a developer to stay aware of relevant information in his work context.

\section{EVALUATION}

Case Study with Professional Developers on jEdit

H1: Composing feeds with information fragments from the developer's work allows her to retrieve relevant items with high precision and recall.

H2: The "Awareness View" supports a developer in finding relevant items.

H3: The composition of dynamic and static fragments provides context that helps the developer interpret the information items. 